analysed using a SOB point-of-care biomarker panel (troponin I, myoglobin, CK-MB, D-dimer, and BNP at the time when patient presented with SOB within 15 minutes of patient's visit to the ED. Thirty-day follow-up for HF, ACS and PE was performed.

The following cut offs were used for determining a positive biomarker:

1. myoglobin $>107 \mathrm{ng} / \mathrm{mL}$,

2. CK-MB $>4.3 \mathrm{ng} / \mathrm{mL}$,

3. troponin I $>0.40 \mathrm{ng} / \mathrm{mL}$,

4. D-dimer $>500 \mathrm{ng} / \mathrm{mL}$, and

5. BNP > $100 \mathrm{ng} / \mathrm{mL}$.

CTCA, CTPA and 2D Echo were taken as the Gold Standard for the diagnosis of ACS, HF and PE.

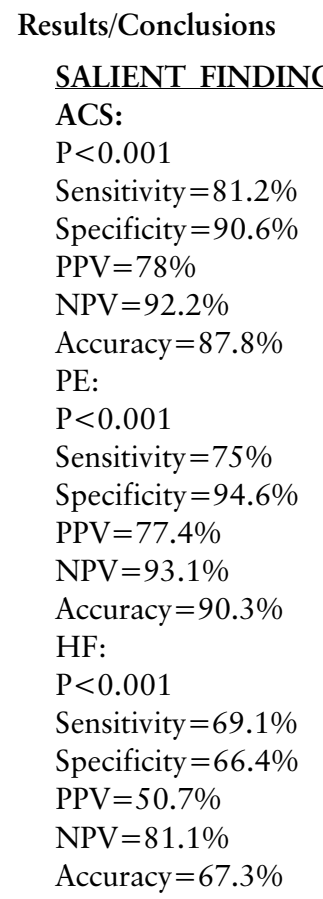

Conclusion We conclude that there is significant correlation between SOB point-of-care biomarker panel diagnosis and final diagnosis.

In patients presenting with $\mathrm{SOB}$, one of the three clinical conditions identified by the SOB point-of-care biomarker panel will lead to earlier diagnosis and initiation of specific management.

\section{POINT OF CARE LUNG ULTRASOUND IN PATIENT TRIAGE: INTEGRATION OF ULTRASOUND INTO A STREAMING PATHWAY FOR COVID-19}

Dominic Craver, Aminah Ahmad, Anna Colclough. Lewisham Hospital

10.1136/emj-2020-rcemabstracts.27

Aims/Objectives/Background Rapid risk stratification of patients is vital for Emergency Department (ED) streaming during the COVID-19 pandemic. Ideally, patients should be split into red (suspected/confirmed COVID-19) and green (non COVID-19) zones in order to minimise the risk of patient-to-patient and patient-to-staff transmission. A robust yet rapid streaming system combining clinician impression with point-of-care diagnostics is therefore necessary.
Point of care ultrasound (POCUS) findings in COVID-19 have been shown to correlate well with computed tomography (CT) findings, and it therefore has value as a front-door diagnostic tool. At University Hospital Lewisham (a district general hospital in south London), we recognised the value of early POCUS and its potential for use in patient streaming.

Methods/Design We developed a training programme, 'POCUS for COVID' and subsequently integrated POCUS into streaming of our ED patients. The training involved Zoom lectures, a face to face practical, a 10 scan sign off process followed by a final triggered assessment. Patient outcomes were reviewed in conjunction with their scan reports.

Results/Conclusions Currently, we have 21 ED junior doctors performing ultrasound scans independently, and all patients presenting to our department are scanned either in triage or in the ambulance. A combination of clinical judgement and scan findings are used to stream the patient to an appropriate area.

Service evaluation with analysis of audit data has found our streaming to be $94 \%$ sensitive and $79 \%$ specific as an indicator of COVID 19. Further analysis is ongoing.

Here we present both the structure of our training programme and our integrated streaming pathway along with preliminary analysis results.

\section{RCEM moderated}

\section{THE EFFECT OF AFFECT: WHAT WE TEACH OUR LEARNERS ABOUT THE ROLE OF PATIENT INDUCED EMOTION IN CLINICAL REASONING IN THE ED AND WHY?}

Susie Roy, Janet Skinner, Alan Jaap. NHS Lothian/University of Edinburgh

\subsection{6/emj-2020-rcemabstracts.28}

Aims/Objectives/Background Few empirical studies explore the contribution of non-clinical factors to perceptions of patient difficulty in EM. Fewer have investigated what students placed in EDs learn about 'difficult' patients or what, if anything, clinicians teach about the topic. We looked to address this. Considering these questions is imperative: patients perceived as frustrating report lower satisfaction with their clinical encounter, experience worse health outcomes and seem to be at risk of medical error secondary to faulty clinical reasoning.

Methods/Design With ethical approval, we undertook three interrelated, qualitative studies to conduct a case study of the undergraduate EM module delivered at Edinburgh University. In the first two, focus groups were used as the method of data collection; five clinician $(n=25)$ and four medical student $(n=21)$ groups were facilitated. In the third, semi-structured interviews with clinicians $(n=12)$ were conducted. All groups/interviews were audio-recorded and transcribed. The data were analysed inductively using reflexive thematic analysis.

Results/Conclusions Frequent attendance, demands, pre-existing relationships and unrealistic expectations contributed to perceived patient difficulty. These were modified by personal and circumstantial factors. Although rarely told, students were aware who these 'difficult' patients were through observing behaviours. Critically, clinicians and students alike believed 